\title{
Which sealant system should I use?
}

\author{
What are the retention rates of resin-based sealants?
}

\author{
Muller-Bolla M, Lupi-Pégurier L, Tardieu C, Velly AM, \\ Antomarchi C. Retention of resin-based pit and fissure \\ sealants: a systematic review. Community Dent Oral Epidemiol \\ 2006; 34:321-336
}

Data sources As well as using Medline, Embase and the Cochrane library to source studies, a search was made by hand of reference lists of the selected articles and dental conference proceedings, and experts were contacted to identify any unpublished research, irrespective of language in each case.

Study selection Two reviewers independently selected the studies. The latter were chosen if randomised, quasi-randomised or controlled clinical trials that used split-mouth design or were parallel group studies, and which compared different available resin-based sealants (RBS) on permanent molars or compared different clinical protocols for the same RBS. Studies comparing RBS with ultraviolet-activated sealants, glass ionomer cements (GIC) or resin-reinforced GIC sealants were not considered and neither were resins indicated for restoration, except for flowable resins.

Data extraction and synthesis Data was extracted by two reviewers independently. Data synthesis was conducted using the program RevMan (version 4.2; Cochrane Collaboration, Dublin, Ireland). Relative risks (RR) were calculated because the outcome was a dichotomous variable; variance was expressed using $95 \%$ confidence intervals $(\mathrm{Cl})$. Metaanalysis was carried out if the studies were homogeneous enough and subgroup analyses were conducted if necessary. The heterogeneity of the results was assessed using chi-squared tests, and sensitivity analyses were performed excluding unpublished studies or poor-quality studies.

Results A total of 31 studies met the inclusion criteria. Sixteen studies compared two different types of RBS, those that were light-cured (LRBS) or auto-polymerised (ARBS), with fluoride-containing sealants (FRBS). Seventy-five per cent were split-mouth design studies. Seven studies compared LRBS with ARBS and nine studies compared LRBS with FRBS. The retention rate of ARBS and LRBS did not differ significantly. LRBS had a significantly higher retention rate than FRBS at 48 months (RR, 0.80; $95 \% \mathrm{Cl}, 0.72-0.89$ ). Only 15 studies of limited quality focused on the best clinical protocol to adopt for sealant application.

Conclusions Only FRBS had a lower retention rate than ARBS or LRBS. This review did not allow the best clinical procedure to be determined because of insufficient studies. Well-designed randomised clinical trials are still needed that focus on sealant retention and consider different clinical procedures, particularly new enamel preparation techniques such as air-abrasion or sono-abrasion.

Address for correspondence: Michèle Muller-Bolla, Faculté de Chirurgie Dentaire, 24 avenue des Diables Bleus, 06357 Nice, France. E-mail: muller@unice.fr.

\section{Commentary}

This is a very welcome systematic review since things are changing rapidly with regard to pit and fissure sealants. The review is also excellent, combining systematic and narrative elements where a systematic approach was not possible because of lack of research evidence. A minor criticism is that, although the article's introduction mentions previous systematic reviews examining these sealants, ${ }^{1-3}$ more description and comparison between these and the present review would have been useful.

Of the 124 clinical trials identified, 31 met the inclusion criteria. Examination of the studies showed that the retention of ARBS and LRBS did not differ significantly at any time point up to 48 months. Also, FRBS had poorer retention than LRBS but no trials compared ARBS with FRBS. The authors suggest that this is because of the presence of fillers in the FRBS: fillers increase the surface tension of the sealant material and therefore reduce the wettability of the material. An analysis comparing autopolymerising and light-cured sealants with and without fillers would have been of value. It is my guess that the unfilled resins would show better retention for the reasons discussed but this needs to be demonstrated. The narrative discussion about the lack of clinical benefit from the presence of fluoride in sealants should be of interest to clinicians.

The review was unable to determine the best clinical technique because of insufficient studies. The statement, "The study comparing mechanical cleaning using pumice with no cleaning does not have any clinical interest because it was proved that both have negative effects on bonding" is not referenced. It should also be noted that the former is the procedure many manufacturers recommend, even though there is the potential for pumice to be left in the fissures. Air abrasion to clean the tooth followed by acid etching was beneficial compared with etching alone but whether it is superior to mechanical cleaning and etching remains to be answered.

It is interesting to note that rubber dam isolation was not more effective than other methods. The use of Er:YAG (erbium-doped yttrium aluminium garnet) laser was not superior to acid etching, and it is also unlikely that dentists would adopt its routine use in sealant placement, for economic and patient-focused reasons, in any case.

The most interesting developments in sealant placement are probably the use of adhesive agents and self-etching systems. The use of hydrophilic primers appears to increase retention. According to the authors, self-etching systems offer as good retention as traditional etching, based on the results of one study. A cohort study, which was excluded for obvious reasons, questions this, ${ }^{4}$ as does a more recent clinical trial. ${ }^{5}$

The conclusions of this review are that the retention of FRBS is inferior to other types of sealant, and many questions remain with regard to clinical technique. 


\section{Chris Deery}

Department of Oral Health and Development, School of Clinical Dentistry, University of Sheffield, Sheffield, UK

1. Llodra JC, Bravo M, Delgado-Rodriguez M, Baca P, Galvez R. Factors influencing the effectiveness of sealants — a meta-analysis. Community Dent Oral Epidemiol 1993 21:261-268.

2. Mejare I, Lingstrom P, Petersson LG, et al. Caries-preventive effect of fissure sealants: a systematic review. Acta Odontol Scand 2003; 61:321-330.
3. Ahovuo-Saloranta A, Hiiri A, Nordblad A, Worthington $\mathrm{H}$, Mäkelä M. Pit and fissure sealants for preventing dental decay in the permanent teeth of children and adolescents. Cochrane Database Systematic Rev 2004, issue 3.

4. Venker DJ, Kuthy RA, Qian F, Kanellis MIJ. Twelve-month sealant retention in school-based program using a self-etching primer/ adhesive. Public Health Dent 2004; 64:191-197.

5. Burbridge L, Nugent Z, Deery C. A randomized controlled trial of the effectiveness of a one-step conditioning agent in sealant placement: 6-month results. Int J Paediatr Dent 2006; 16:424-430.

Evidence-Based Dentistry (2007) 8, 8-9. doi:10.1038/sj.ebd.6400462

Key to evidence graphic used in the Evidence-based Dentistry Journal

The graphic is based on the Centre for Evidence-based Medicine levels of Evidence tables www.cebm.net/levels_of_evidence.asp (see Evidence-based Dentistry 2003;4: p 17-18)

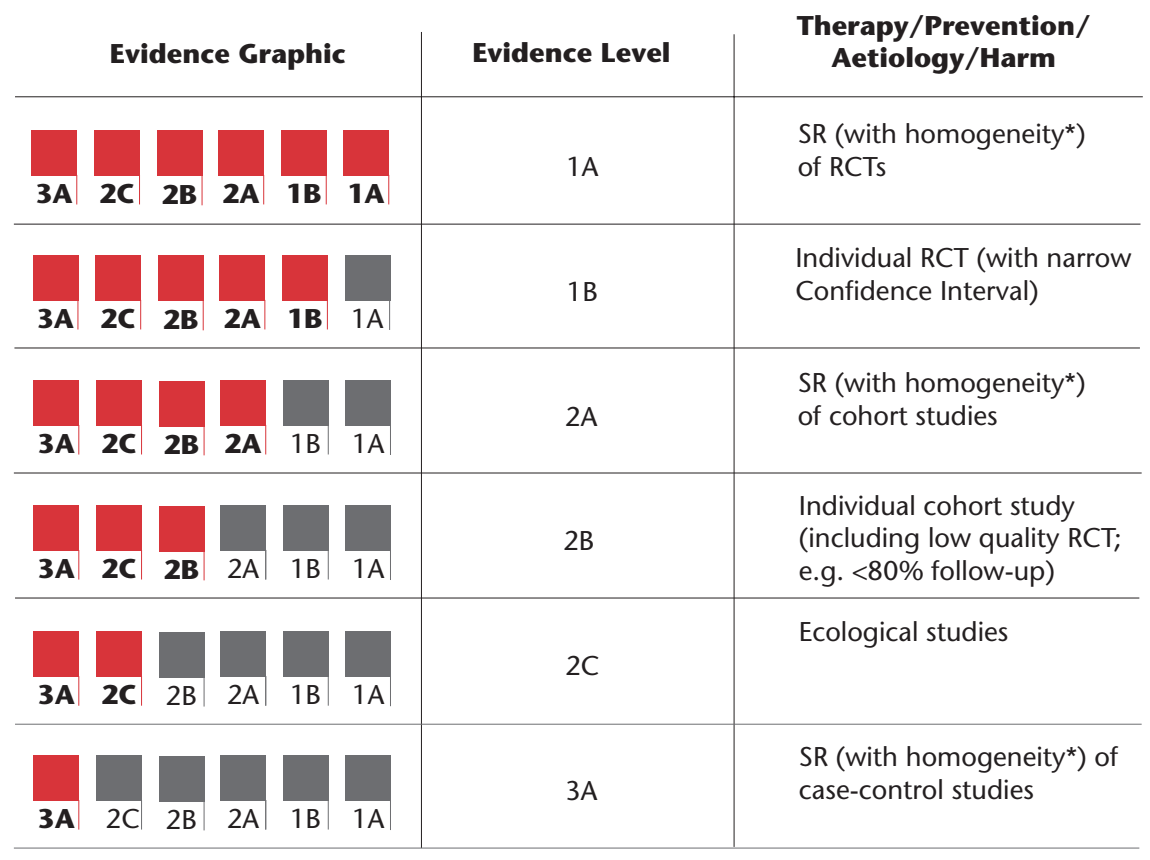

* By homogeneity we mean a systematic review that is free of worrisome variations (heterogeneity) in the directions and degrees of results between individual studies. Not all systematic reviews with statistically significant heterogeneity need be worrisome, and not all worrisome heterogeneity need be statistically significant. 